\title{
On the Dominator Colorings in Bipartite Graphs
}

\author{
Ralucca Gera, * \\ Department of Applied Mathematics \\ Naval Postgraduate School \\ Monterey, CA 93943, USA
}

\begin{abstract}
A graph has a dominator coloring if it has a proper coloring in which each vertex of the graph dominates every vertex of some color class. The dominator chromatic number $\chi_{d}(G)$ is the minimum number of color classes in a dominator coloring of a graph $G$. In this paper we study the dominator chromatic number for the hypercube, $Q_{n}=Q_{n-1} \times K_{2}$ (with $Q_{1} \cong P_{2}, n \geq 2$ ), and more generally for bipartite graphs. We then conclude it with open questions for further research.
\end{abstract}

\section{Introduction}

Interconnection networks are relevant for parallel computer systems and have been studied extensively lately. Examples of popular interconnection networks are tree, mesh, hypercube, and star graph. The hypercube of dimension $n, Q_{n}$, (also known as $n$-cube, or binary cube) is a type of parallel computer with the processors linked together in the shape of a hypercube. We present bounds on the domination chromatic number of $Q_{n}$ in terms of the domination number and the dominator chromatic number of $Q_{n-1}$. We also give sharp bounds for the dominator chromatic number of bipartite graphs.

An interconnection network can be represented as an undirected graph where a processor is represented as a vertex, and communication channels between processors are the edges between corresponding vertices.

For every positive integer $n$, the hypercube of dimension $n$ is defined to be the graph whose vertex set is the set of all $n$-dimensional Boolean vectors. For example, vertices are labeled with vectors having $n$ coordinates (each of which is 0 or 1 ), and then edges are the pairs of vertices whose $n$-vectors differ in exactly one coordinate. A slightly different definition of the hypercube of dimension

* Research supported by the Research Initiation Program Grant at the Naval Postgraduate School. $n$ which is more useful for this paper, is a recursive definition: $Q_{n}=Q_{n-1} \times K_{2}$, with $Q_{1} \cong P_{2}$. Recall that $\left|V\left(Q_{n}\right)\right|=2^{n}, n \geq 1$. The hypercube $Q_{n}$ is an $n$-regular bipartite graph.

A dominating set $S$ is a subset of the vertices in a graph such that every vertex in the graph either belongs to $S$ or has a neighbor in $S$. The domination number, $\gamma(G)$, is the order of a minimum dominating set. The topic has long been of interest to researchers [6, 7]. This definition also leads naturally to the associated optimization problem, which is to find a dominating set of minimum cardinality. Numerous variants of this problem have been studied [2, 6, 7, 9], and here we study one more that was introduced in [5]. The associated decision problem, DOMINATING SET, occupies a prominent place in the computational complexity literature. Finding a minimum DOMINATING SET is NP-complete on arbitrary graphs [4].

A proper coloring of a graph $G=(V(G), E(G))$ is a function from the vertices of the graph to a set of colors such that any two adjacent vertices have different colors. The chromatic number is the minimum number of colors needed in a proper coloring of a graph. Graph coloring is used as a model for a vast number of practical problems involving allocation of scarce resources (e.g., scheduling problems), and has played a key role in the development of graph theory and, more generally, discrete mathematics and combinatorial optimization. Graph $k$-colorability is NP-complete in the general case, although the problem is solvable in polynomial time for many classes [4].

A dominator coloring of a graph $G$ is a proper coloring in which each vertex of the graph dominates every vertex of some color class. The dominator chromatic number $\chi_{d}(G)$ is the minimum number of color classes in a dominator coloring of a graph $G$. A $\chi_{d}(G)$-coloring of $G$ is any dominator coloring with $\chi_{d}(G)$ colors. Our study of this problem is motivated by [3], [5], and [8]. Finding a minimum DOMINATING COLORING SET is NP-complete on arbitrary graphs [5]. A number of problems that are NP-complete in general are polynomially solvable on various classes of perfect graphs, so it seems natural to find bounds or optimal solutions for these classes. A graph $G$ is perfect if $\chi(H)=\omega(H)$ for all induced subgraphs $H$ of $G$, where 
$\omega(H)$ is the order of the largest complete subgraph in $H$. One particular perfect class is the bipartite class.

In this paper we will only be working with connected graphs. For further information on the dominator chromatic number in graphs (and in particular for disconnected graphs), readers should research [5]. We begin with preliminary information leading to the bounds of the chromatic number of the $n$-cube in Section 2 . We then study chromatic colorings in bipartite graphs in Section 3 and 4, and conclude with further open research in Section 5 .

\section{A bound on dominator chromatic number of hypercube}

We begin our study with an example of finding the dominator chromatic number for the graph in Figure 1 in order to show an approach in finding the dominator chromatic number of a graph.

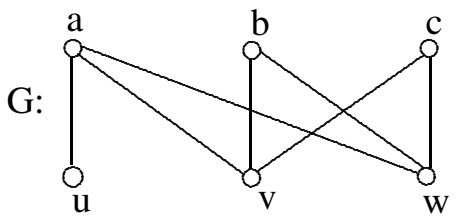

Figure 1: An example

Example: We show that the bipartite graph $G$ in Figure 1 has $\chi_{d}(G)=3$. Since $G$ is a bipartite graph, at least two colors are needed to obtain a proper coloring of the graph, namely vertices $a, b$, and $c$ should receive one color, and vertices $u, v$, and $w$ should receive a different one. Note that this coloring is unique up to isomorphism. However then, the vertex $u$ does not dominate a color class since it is not the only one of its color class (so it cannot dominate its own class), and also it is not adjacent to all vertices in the other color class (thus not dominating the other color class either). Therefore, at least 3 colors are needed for the graph, and so $\chi_{d}(G) \geq 3$.

Now, define a coloring where the vertices $a, b$, and $c$ receive color 1 , vertex $u$ receives color 2 , and vertices $v$ and $w$ receive color 3 . Since each of the vertices $a, b$, and $c$ dominate the color class 3 , the vertex $u$ dominates the color class 2 , and each of the vertices $v$ and $w$ dominate the color class 1 , it follows that 3 colors will suffice, and so $\chi_{d}(G) \leq 3$. Therefore $\chi_{d}(G)=3$.

We now describe an algorithm that gives a dominator coloring of the hypercube $Q_{n+1}$, in terms of the dominator chromatic number and the domination number of the hypercube $Q_{n}$.
Proposition 2.1 Let $Q_{n}$ be the $n$-cube of dimension $n \geq 2$. Then

$$
\chi_{d}\left(Q_{n+1}\right) \leq \chi_{d}\left(Q_{n}\right)+\gamma\left(Q_{n}\right),
$$

with $\chi_{d}\left(Q_{2}\right)=2$.

Proof. Since $Q_{n}$ is bipartite, we can properly color $Q_{n}$ by alternating the colors $a$ and $b(a \neq b)$, in such a way that no two adjacent vertices will have the same color. Such a coloring is unique up to isomorphism. Now assign colors $1,2, \ldots, \gamma\left(Q_{n}\right)$ to the vertices in a minimum dominating set $S$ of $Q_{n}$, and leaving the rest of the vertices colored with $a$ and $b$ as before. This is a dominator coloring of $Q_{n}$ since it is still a proper coloring, and the dominating set provides the color class that every vertex dominates.

We now show how we can obtain a dominator coloring of $Q_{n+1}$ from a dominator coloring of $Q_{n}$ and the dominating set of $Q_{n}$. Recall that we obtain $Q_{n+1}$ from two copies of $Q_{n}$, say $Q^{\prime}$ and $Q^{\prime \prime}$ by adding $2^{n}$ more edges between the corresponding vertices of the two copies $Q^{\prime}$ and $Q^{\prime \prime}$. We then define a coloring $c^{\prime}$ of $Q_{n+1}$ by (1) keeping the coloring of $Q^{\prime}$ as defined above for $Q_{n}$, and (2) changing the coloring for $Q^{\prime \prime}$ from the one of $Q_{n}$ as follows: interchange the colors $a$ and $b$, and replace the color $i$ $\left(1 \leq i \leq \gamma\left(Q_{n}\right)\right)$ by the color $\gamma\left(Q_{n}\right)+i$. This new coloring $c^{\prime}$ uses $\chi_{d}\left(Q_{n}\right)+\gamma\left(Q_{n}\right)$ colors.

Claim: The coloring $c^{\prime}$ is a dominator coloring of $Q_{n+1}$. We first show that $c^{\prime}$ is a proper coloring. We only need to worry about the vertices color $a$ or $b$ since the others get non-repeated colors. Let $x^{\prime \prime} \in Q^{\prime \prime}$ be the corresponding vertex to $x^{\prime} \in Q^{\prime}$. Recall that (1) a proper coloring of $Q^{\prime}$, (2) a proper coloring of $Q^{\prime \prime}$, and (3) if $x^{\prime}$ is colored $a$ or $b$, then $x^{\prime \prime}$ is colored $b$ or $a$, respectively, as the coloring of $Q^{\prime \prime}$ interchanges the colors $a$ and $b$. So $c^{\prime}$ is a proper coloring.

On the other hand, since the vertices colored $1,2 \ldots, \gamma\left(Q_{n}\right), \ldots 2 \gamma\left(Q_{n}\right)$ form a dominating set of $Q_{n+1}$, it follows that each vertex colored $j\left(1 \leq j \leq 2 \gamma\left(Q_{n}\right)\right)$ dominates itself, and each vertex colored $a$ or $b$ dominates the class $j$ of the vertex that it is adjacent to in the dominating set $S$. Therefore $c^{\prime}$ is a dominator coloring of $Q_{n+1}$, and the result follows.

We now show the above theorem for $Q_{3}$ and $Q_{4}$ :

$$
\chi_{d}\left(Q_{3}\right) \leq \chi_{d}\left(Q_{2}\right)+\gamma\left(Q_{2}\right),
$$

and

$$
\chi_{d}\left(Q_{4}\right) \leq \chi_{d}\left(Q_{3}\right)+\gamma\left(Q_{3}\right)
$$

First, note that the dominator chromatic number of $Q_{2}$ is 2 , and its domination number is 2 , so by Theorem 2.1

$$
\chi_{d}\left(Q_{3}\right) \leq \chi_{d}\left(Q_{2}\right)+\gamma\left(Q_{2}\right)=4,
$$

as it can be seen in Figure 2 (the filled in vertices in Figure 2 form a minimum dominating set, and colors $a, b, 1$ and 2 show $\left.\chi_{d}\left(Q_{3}\right)=4\right)$. 


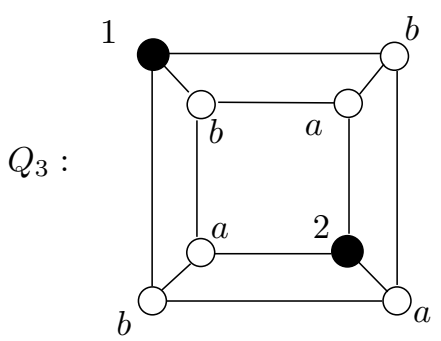

Figure 2: $\chi_{d}\left(Q_{3}\right) \leq 4$

Now, the dominator chromatic number of $Q_{3}$ is at most 4 , and its domination number is 2 , and so by Theorem 2.1

$$
\chi_{d}\left(Q_{4}\right) \leq \chi_{d}\left(Q_{3}\right)+\gamma\left(Q_{3}\right) \leq 6,
$$

(the filled in vertices in Figure 3 form a minimum dominating set, and colors $a, b, 1,2,3$ and 4 show $\left.\chi_{d}\left(Q_{3}\right)=4\right)$.

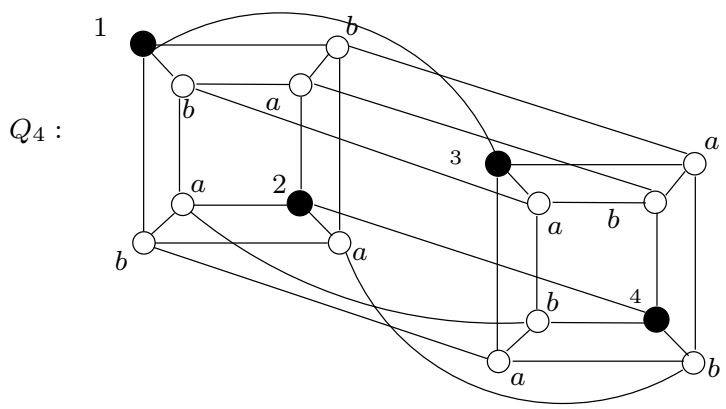

Figure 3: $\chi_{d}\left(Q_{4}\right) \leq 6$

We can obtain the dominator coloring of $Q_{4}$ from the one of $Q_{3}$ as follows: Let $Q^{\prime}$ and $Q^{\prime \prime}$ be two copies of $Q_{3}$ with their respective dominator colorings. We define a coloring of $Q_{4}$ from the dominator coloring of the two copies $Q^{\prime}$ and $Q^{\prime \prime}$, by (1) keeping the coloring of $Q^{\prime}$ as the coloring of $Q_{3}$, and (2) changing the coloring of $Q^{\prime \prime}$ from the coloring of $Q_{3}$ as follows: interchange the colors $a$ and $b(a \neq b)$, and replace the color $i\left(1 \leq i \leq \gamma\left(Q_{3}\right)=2\right)$ by the color $\gamma\left(Q_{3}\right)+i$

\section{Bounds for the dominator chro- matic number for bipartite graphs}

Recall that $Q_{n}$ is a type of bipartite graph. We now study dominator coloring in bipartite graphs starting with general bounds in terms of the order of the graph, and some characterizations.
Theorem 3.1 Let $G$ be a connected bipartite graph. Then

$$
2 \leq \chi_{d}(G) \leq\left\lfloor\frac{n}{2}\right\rfloor+1
$$

The bounds are sharp.

Proof. Let $G$ be a connected bipartite graph. Since every dominating coloring must be a proper coloring, and $\chi(G)=$ 2 , it follows that $\chi_{d}(G) \geq 2$. For the upper bound, let $V_{1}$ and $V_{2}$ be the two bipartite sets of $G$, with $\left|V_{1}\right| \leq\left|V_{2}\right|$. Then the coloring that assigns colors $1,2, \ldots,\left|V_{1}\right|$, to the vertices of $V_{1}$, and color $\left|V_{1}\right|+1$ to the vertices of $V_{2}$ is a dominator coloring where the vertices of $V_{1}$ provide the color classes that vertices dominate since $G$ is connected. Thus $\chi_{d}(G) \leq\left|V_{1}\right|+1$, and since $\left|V_{1}\right| \leq\left\lfloor\frac{n}{2}\right\rfloor$, the result follows.

Now, for sharpness of the bounds, note that the complete bipartite graph $K_{a, b}(a, b \geq 2)$ has a dominator chromatic number of 2 , and so the lower bound is sharp. As for the upper bound, consider the corona of the path $P_{n}, \operatorname{Cor}\left(P_{n}\right)$, which is the graph obtained from $P_{n}$ by attaching a pendant to each vertex of the path. Since each pendant or its stem has to belong to a dominating set, it follows that the vertices of the path form a minimum dominating set of $\operatorname{Cor}\left(P_{n}\right)$, so $\gamma\left(\operatorname{Cor}\left(P_{n}\right)\right)=n$. Also, the coloring that assigns colors $1,2, \ldots, n$ to the vertices of $P_{n}$, and color $n+1$ to the independent vertices that form the set $V\left(\operatorname{Cor}\left(P_{n}\right)\right)-V\left(P_{n}\right)$ is a minimum dominator coloring. To see that, observe that each pendant either dominates its own color class (if the pendant is the only one of that color), or it dominates the color class of its stem (if the stem is the only one of that color). And so at least $n$ vertices must receive their unique color, and at least one more color is needed for the rest of the vertices. And so $\chi_{d}\left(\operatorname{Cor}\left(P_{n}\right)\right)=\left\lfloor\frac{n}{2}\right\rfloor+1$.

We next show that all the values in between 2 and $\left\lfloor\frac{n}{2}\right\rfloor+1$ can be attained as the dominator chromatic number of some bipartite graph of order $n$.

Proposition 3.2 Let $k$ be an integer with $2 \leq k \leq\left\lfloor\frac{n}{2}\right\rfloor+1$. Then there is a connected bipartite graph $G$ with dominator chromatic number $k$ and of order $n$.

Proof. Consider the graph $G$ obtained from the path $P_{k}$ with $V\left(P_{k}\right): v_{1}, v_{2}, \ldots, v_{k}$, by adding the vertices $u_{1}, u_{2}, \ldots, u_{k}$ so that $u_{i} v_{i} \in E(G)$ for all $i(1 \leq i \leq k)$, and by adding $n-2 k$ vertices $u_{j}(0 \leq j \leq n-2 k)$, to the vertex $v_{k}$ (see Figure 4 , where the filled in vertices form a minimum dominating set).

Then the set $v_{1}, v_{2}, \ldots, v_{k}$ form a minimum dominating set since for each pendant $u_{i}(1 \leq i \leq k)$ either $v_{i}$ or $u_{i}$ must belong to any dominating set. Thus $\gamma(G)=k$.

On the other hand each pendant $u_{i}(1 \leq i \leq k)$ either dominates its own color class (if $u_{i}$ is the only one of that 

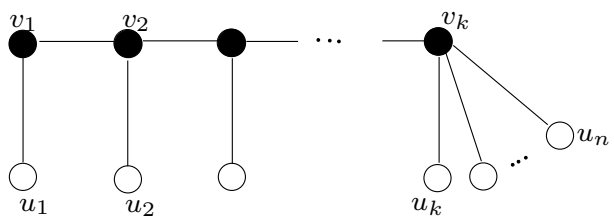

Figure 4: The realization graph

color), or it dominates the color class of $v_{i}$ (if $v_{i}$ is the only one of that color). And so at least $k$ vertices must receive their unique color, and at least one more color is needed for the rest of the vertices. Thus $\chi_{d}(G) \geq k$. Also since the coloring that assigns color $i(1 \leq i \leq k)$ to the vertex $v_{i}$, and color $k+1$ to the rest of the vertices is a dominator coloring with $k+1$ colors, we obtain that $\chi_{d}(G) \leq k+1$.

Now we present a characterization of the lower bound.

Proposition 3.3 Let $G$ be a bipartite graph of order at least 2. Then

$$
\chi_{d}(G)=2 \text { if and only if } G=K_{m, n} .
$$

Proof. If $G=K_{m, n}$, then the coloring that assigns one color to one bipartite set and another color to the other bipartite set is a dominator coloring, and so $\chi_{d}(G)=2$. Thus assume that $\chi_{d}(G)=2$ for some bipartite graph $G$. Then, up to isomorphism, there is a unique dominator coloring that assigns color 1 to the bipartite set $V_{1}$, and color 2 to the bipartite set $V_{2}$. We wish to show that we have a complete graph. We consider two cases.

Case 1. $\left|V_{i}\right|=1$ for either $i=1$ or $i=2$. Then $G=$ $K_{1, n}$, which is a complete bipartite graph. Thus, assume that both $\left|V_{i}\right| \geq 2$, for $i=1,2$.

Case 2. $\left|V_{i}\right| \geq 2$ for $i=1$ or $i=2$. Let $x \in V_{1}$. Since $x$ cannot dominate the color class of $V_{1}$, it follows that $x$ dominates all the vertices in $V_{2}$. Similarly, for some vertex $y \in V_{2}, y$ must be adjacent to all the vertices of $V_{1}$, and the result follows.

For the upper bound we have a necessary condition.

Proposition 3.4 Let $G$ be a bipartite graph. Then $\operatorname{Cor}(G)$ is a bipartite graph whose chromatic domination number is $\left\lfloor\frac{|\operatorname{Cor}(G)|}{2}\right\rfloor+1$.

Proof. Let $G$ be a bipartite graph of order $n$, and define $G^{\prime}$ to be the corona of the graph $G$, i.e. the bipartite graph $G$ with a pendant to each vertex of the graph. Note that $G^{\prime}$ is bipartite. We now show that $\chi_{d}\left(G^{\prime}\right)=\left\lfloor\frac{\left|G^{\prime}\right|}{2}\right\rfloor+1$. We define a coloring of $G^{\prime}$ by assigning colors $1,2, \ldots,|G|$ to all vertices of the graph $G$, and color $|G|+1$ to all the pendants. This is a dominator coloring since every vertex in $G$ dominates itself, and each leaf dominates the stem that it is adjacent to. Also, this is a minimum dominator coloring since at least one new color is needed for every stem-vertex pair, and at least one other color for the rest of the vertices.

Now this is not a sufficient and necessary condition since $C_{4}+e$ satisfies the relation $\chi_{d}\left(C_{4}+e\right)=\left\lfloor\frac{\left|C_{4}+e\right|}{2}\right\rfloor+1$.

\section{The dominator chromatic number of bipartite graphs in terms of domination number}

We find bounds for connected bipartite graphs in terms of the domination number of the graph.

Theorem 4.1 Let $G$ be a bipartite graph. Then

$$
\gamma(G) \leq \chi_{d}(G) \leq 2+\gamma(G)
$$

Proof. Let $c$ be a minimum dominator coloring of $G$ with colors $1,2, \ldots, \chi_{d}(G)$. For each color class of $G$, let $x_{i}$ be a vertex in the color class $i$, with $1 \leq i \leq \chi_{d}(G)$. Consider the set $S=\left\{x_{i}: 1 \leq i \leq \chi_{d}(G)\right\}$. We show that $S$ is a dominating set. Let $x \in V(G)$. Since each vertex of $G$ must dominate a color class, and since domination is reflexive, it follows that $x$ is dominated by all vertices of some color class of $G$. Since $S$ contains one vertex of each color class, we have that all vertices of $G$ are dominated by some vertex in $S$. Thus the domination number is a lower bound for the dominator chromatic number in the graph.

For the upper bound, since the chromatic number of the bipartite graph $G$ is two, it follows that we can color the vertices of $G$ by alternating two colors, say $a$ and $b(a \neq b)$, so that no two adjacent vertices will share the same color. Now, define a coloring $c$ (which we will show to be a dominator coloring) of $G$ by assigning colors $1,2, \ldots, \gamma(G)$ to the vertices in a minimum dominating set $S$ of $G$, and leaving the rest of the vertices colored with $a$ and $b$ as before. Note that this is a dominator coloring of $G$ since (1) it is a proper coloring as no two adjacent vertices share the same colors, and (2) the vertices of the dominating set provide the color classes that every vertex of $G$ dominates (see Figure 2 or 3 for example).

We define a bipartite graph to be a graph of class 0 if $\chi_{d}(G)=\gamma(G)$, of class 1 if $\chi_{d}(G)=\gamma(G)+1$, and also of class 2 if $\chi_{d}(G)=\gamma(G)+2$.

We now show some examples for each class and results. Let the graph $K_{m, n}$ be the complete bipartite graph with $m, n \geq 2$, the graph $K_{1, a}$ be the star with $a$ pendants, the graph $S_{a, b}$ be the double star with $a+b$ pendants $(a, b, \geq 2)$, 
and $P_{n}$ be the path on $n \geq 3$ vertices. There are classes of graphs for which $\chi_{d}(G) \in\{\gamma(G), \gamma(G)+1, \gamma(G)+2\}$, so the bounds above are sharp, and every value in between is attainable. To see this, for example,

$G$ is of class $\quad \begin{cases}0 & \text { for } G=K_{n, m}, \\ 1 & \text { for } G=K_{1, a} \text { or } S_{a, b} \\ 2 & \text { for } P_{n} \text { for } n \geq 3 .\end{cases}$

For the dominator chromatic number of standard classes of graphs, the interested reader should consult [5]. Besides the classes mentioned above there are a few more subclasses that fit in each category. As proved in [5], the class of connected caterpillars for which the vertices of degree 2 or less are independent are of class 1 . Spider graphs are also of class 1 . Next we provide a method of obtaining a graph of class 1 , having the original graph induced.

Proposition 4.2 Let $G$ be a bipartite graph. Then there is always a graph of class 1 that contains $G$ as an induced graph.

Proof. Let $G$ be a bipartite graph of order $n$, and define $G^{\prime}$ to be the corona of the graph $G$. As shown in Proposition corona, the result follows.

As it may seem, most of the graphs are of class 1 or 2 . However, there is an infinite family of graphs of class 0 , that includes the complete bipartite graphs. First, recall that the open neighborhood of a vertex $x \in V(G)$ is $N(x)=$ $\{y \in V(G): x y \in E(G)\}$, and the closed neighborhood of $x$ is $N[x]=N(x) \cup\{x\}$. Similarly, for a set $X \in$ $V(G)$, recall that the open neighborhood of the set $X$ is $N(X)=\{y \in V(G): x y \in E(G), \exists x \in X\}$, and the closed neighborhood of the set $X$ is $N[X]=N(X) \cup\{x\}$.

We define a bipartite graph $G$ to be partially-complete bipartite if it has the following structure: $G$ is obtained from $\alpha$ disjoint copies of complete bipartite graphs $K_{a_{i}, b_{i}}$ where $a_{i}, b_{i} \geq 3(i \geq 1)$, by adding edges between $K_{a_{i}, b_{i}}$ and $K_{a_{j}, b_{j}}(i \neq j \geq 1)$, with the condition that either

(1) for each partite set $V_{i}$ of $K_{a_{i}, b_{i}}$, there is no set $X \subseteq$ $N\left(V_{i}\right)$ such that for every $V_{j}(i \neq j),\left|N\left(V_{j}\right) \cap X\right|=$ 1, and also, $\forall v_{i} \in V_{i}, \exists u_{i} \in X$ with $u_{i} v_{i} \in E(G)$, or

(2) for each partite set $V_{i}$ of $K_{a_{i}, b_{i}}$, there is no set $X \subseteq$ $V_{i}$ such that if the $j_{\ell}$ bipartite sets $V_{j_{1}}, V_{j_{2}}, \ldots, V_{j_{\ell}} \subseteq$ $N(X)$, then $j_{\ell} \geq|X|$.

The above definition allows the addition of a limited number of edges in between the complete bipartite subgraphs by prohibiting either (1) for some $i$ there is a set composed of at most one vertex of each $K_{a_{j}, b_{j}}$ that can dominate one of the bipartite sets of $K_{a_{i}, b_{i}}(i \neq j)$, or (2) for some $i$ there is a set $V_{i}$ within a bipartite subgraph that can dominate $\left|V_{i}\right|+1$ bipartite sets of different disjoint bipartite subgraphs.

An example is shown in Figure 5, where the numbers represent the dominator color classes and the filled in vertices form a minimum dominating set.

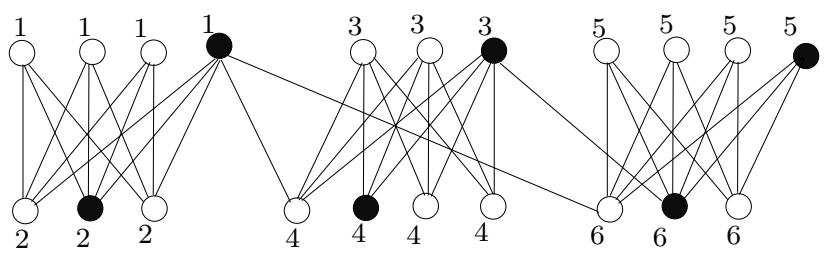

Figure 5: A graph of class 0 .

An example that is not of class 0 (because there is a set $X$ with the property described above) is provided in Figure 6, where again the numbers represent the dominator color classes and the filled in vertices form a minimum dominating set. Note that the domination number in Figure 6 is one less than the dominator chromatic number.

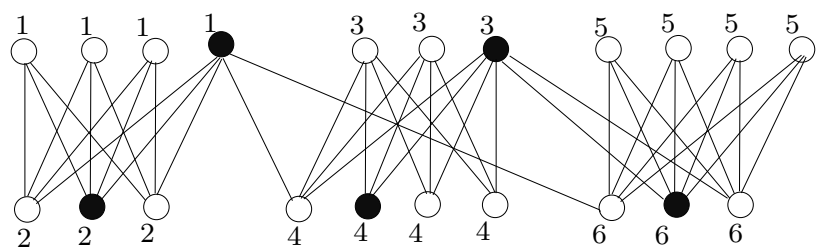

Figure 6: Not a graph of class 0 .

We next show that the partially-complete bipartite graphs are the only bipartite graphs of class 0 .

Proposition 4.3 Let $G$ be a bipartite graph of order at least 4. Then

$$
\begin{gathered}
\chi_{d}(G)=\gamma(G) \text { if and only if } G \text { is a partially-complete } \\
\text { bipartite graph. }
\end{gathered}
$$

Proof. Let $G$ be partially-complete bipartite graph. Then $G$ contains $\alpha$ disjoint complete bipartite graphs $K_{a_{i}, b_{i}}$ $(1 \leq i \leq \alpha)$. We show that $\chi_{d}(G)=\gamma(G)=2 \alpha(\alpha \geq 1)$. Since each bipartite set of each copy of $K_{a_{i}, b_{i}}$ has at least 3 vertices, it follows that either (1) at least one vertex of each bipartite set, or (2) at least 3 vertices of each complete bipartite graph must belong to a dominating set of $G$. Since a set that contains one arbitrary vertex of each bipartite graph is a dominating set, we have that $\gamma(G)=2 \alpha$. By Theorem 3.1, $\chi_{d}(G) \geq \gamma(G)=2 \alpha$, and since the coloring that assigns a unique color to each bipartite set of each bipartite subgraph is a dominator coloring, it follows that $\chi_{d}(G)=\gamma(G)=2 \alpha$. 
For the converse, assume that $\chi_{d}(G)=\gamma(G)=k(2 \leq$ $k \leq \frac{n}{2}+2$ ) for some bipartite graph $G$.

Let the two bipartite sets of $G$ be $V_{1}$ and $V_{2}$. Note that in a dominator coloring of $G$ we cannot have every color appear in both bipartite sets since then it is impossible for the vertices of the graph to dominate some color class. So assume that colors $1,2, \ldots, s$ are only used in one bipartite set, either $V_{1}$ or $V_{2}$, and colors $s, s+1, \ldots, k$ alternate between $V_{1}$ and $V_{2}$ on nonadjacent vertices.Let $v$ be a vertex in the color class $t(s \leq t \leq k)$. Since color class $t$ appears both in $V_{1}$ and in $V_{2}$, it follows that $v$ has to be adjacent to all the vertices of some color class $p$, for some $p(1 \leq p \leq s-1)$. Also, every vertex in color class $p$ $(1 \leq p \leq s-1)$ either dominates its own class (if it is the only vertex in that color class), or it dominates some other class $r(1 \leq r \leq s-1)$ (being adjacent to all the vertices in color class $r$ ). And so, choosing one vertex in color class $r$ for all $r(1 \leq r \leq s-1)$, we obtain a dominating set of size $s-1$. Since $\gamma(G)=\chi_{d}(G)$, it follows that each color appears just in one bipartite set, and each vertex is either (1) the only one in its color class, or (2) it is adjacent to all vertices of a different color class. Thus $G$ is partially-complete bipartite graph.

As a corollary of Propositions 4.1 and 4.3 we have the following.

Corollary 4.4 Let $G$ be a tree. Then

$$
\gamma(G)+1 \leq \chi_{d}(G) \leq \gamma(G)+2
$$

Note that $\chi_{d}(G)=2+\gamma(G)$ for $P_{n}$, for $n \geq 8$, and $\chi_{d}(G)=1+\gamma(G)$ for $\alpha$ copies of stars identified at one pendant. Thus both values are attained. Moreover, for any tree $T$, if there is a minimum dominating set $S$ such that $V(T)-S$ is an independent set, then $\chi_{d}(T)=\gamma(T)+1$.

The following is a corollary of Propositions 4.1 and 4.3.

Corollary 4.5 Let $Q_{n}$ be the $n$-cube of dimension $n \geq 3$. Then

$$
\gamma\left(Q_{n}\right)+1 \leq \chi_{d}\left(Q_{n}\right) \leq \gamma\left(Q_{n}\right)+2 \text {. }
$$

We actually conjecture the following about the hypercube.

Conjecture 4.6 Let $Q_{n}$ be the $n$-cube of dimension $n \geq 4$. Then

$$
\chi_{d}\left(Q_{n}\right)=2+2^{n-2} .
$$

\section{Further Research}

We conclude the paper with a few questions for further research.
The $n$-dimensional star graph (see [1]) has been recognized as an attractive alternative to the hypercube, and so it would be an interesting class to be studied. An $n$ dimensional star graph $S_{n}$, is a graph whose (1) vertices are $n$-arrangements of the numbers $1,2, \ldots, n$ (where the arrangement 123 is not the same as the arrangement 231 for example), and (2) edges are placed between vertices $x$ and $y$ if given the label of $x$, say $a_{1} a_{2} \ldots a_{n}$, we obtain the label of $y$ by swapping $a_{1}$ with some $a_{i}(2 \leq i \leq n)$, i.e. the label of $y$ is of the form $a_{i} a_{2} \ldots a_{i-1} a_{1} a_{i+1} \ldots a_{n}$. Note that this is a different definition than the graph theoretical definition of stars.

Question 1: Is there a characterization for each class defined above?

Question 2: Can the conjecture on the dominator chromatic number be proved?

\section{Acknowledgment}

The author would like to thank Steve Horton for many stimulating discussions and for reading drafts of this paper.

\section{References}

[1] S. B. Akers, D. Harel, and B. Krishnameurthy, The star graph: An attractive alternative to the $n$-cube, International Conference on Parallel Processing, pages 393-400, 1987.

[2] C. Berge, Theory of Graphs and its Applications, no. 2 in Collection Universitaire de Mathematiques, Dunod, Paris, 1958.

[3] E. Cockayne, S. Hedetniemi, and S. Hedetniemi, Dominating partitions of graphs, tech. rep., 1979. Unpublished manuscript.

[4] M. R. Garey and D. S. Johnson, Computers and Intractability, W. H. Freeman and Co., 1978.

[5] R. Gera, S Horton, C. Rasmussen, Dominator Colorings and Safe Clique Partitions, Congressus Numerantium (2006).

[6] T. W. Haynes, S. T. Hedetniemi, and P. J. Slater, Domination in Graphs: Advanced Topics, Marcel Dekker, New York, 1998.

[7] — Fundamentals of Domination in Graphs, Marcel Dekker, New York, 1998.

[8] S. M. Hedetniemi, S. T. Hedetniemi, and A. A. McRae, Dominator colorings of graphs, (2006). in preparation.

[9] O. Ore, Theory of Graphs, no. 38 in American Mathematical Society Publications, AMS, Providence, 1962. 